\title{
Trafficking of Methicillin-Resistant Staphylococci and Co-Colonization with Vancomycin-Resistant Enterococci
}

\author{
Eman J. Sharaf ${ }^{a}$ Abiola C. Senok ${ }^{b}$ Edet E. Udo ${ }^{c}$ Giuseppe A. Botta ${ }^{a, d}$ \\ ${ }^{a}$ Department of Microbiology, Immunology and Infectious Diseases, Arabian Gulf University, Manama, Bahrain; \\ ${ }^{b}$ Department of Clinical Sciences, College of Medicine, University of Sharjah, Sharjah, United Arab Emirates; \\ 'Department of Microbiology, Faculty of Medicine, Health Sciences Centre, Kuwait University, Jabriya, Kuwait; \\ ${ }^{\mathrm{d}}$ Department of Medical and Morphological Research, Section of Microbiology, Medical School, Udine, Italy
}

\section{Key Words}

Methicillin-resistant Staphylococcus aureus •

Methicillin-resistant coagulase-negative staphylococci •

Vancomycin-resistant enterococci

\begin{abstract}
Objectives: To determine the trafficking of methicillin-resistant staphylococci between the hospital and community as well as the occurrence of co-colonization with vancomycinresistant enterococci (VRE). Subjects and Methods: From November 2005 to April 2006, methicillin-resistant Staphylococcus aureus (MRSA) and methicillin-resistant coagulasenegative Staphylococcus (MRCoNS)-positive patients at the Salmaniya Medical Complex, Bahrain were assessed for VRE co-colonization. Characterization of vancomycin resistance genotype by PCR was carried out. Close family contacts were screened for MRSA and pulsed-field gel electrophoresis (PFGE) analysis of MRSA isolates from patient-family member pairs was conducted. Results: One hundred and eightytwo patients (93 MRSA; 89 MRCoNS) and 356 family members were enrolled. Seven MRSA and 41 MRCoNS strains were isolated from the family members. PFGE analysis re-
\end{abstract}

vealed the presence of variants of a single MRSA clone among patients and their relatives. A total of 112 patients (62 MRSA; 50 MRCoNS) provided stool for VRE screening. Of these 13 stool specimens (11.6\%) were VRE-positive. All the VRE isolates were from MRSA-positive patients, thus positivity rate among MRSA patients was $20.9 \%(n / N=13 / 62)$. These were predominantly Enterococcus gallinarum with vanC1 genotype and one strain was Enterococcus faecium (vanB genotype). Two E. gallinarum isolates harbored an additional vanB gene. The majority of VRE isolates were from patients in medical and surgical units ( $\mathrm{n} / \mathrm{N}=10 / 13 ; 77 \%)$. Male gender, prolonged hospitalization and presence of co-morbidities were significantly associated with MRSA/VRE co-colonization $(p<0.05)$. Conclusion: MRSA/VRE co-colonization with MRSA trafficking between the hospital and community environment is a public health concern occurring in our setting.

Copyright $\odot 2011$ S. Karger AG, Basel

Presented in part as a poster at the 18th European Congress of Clinical Microbiology and Infectious Diseases, Barcelona, 19-22 April 2008.

\section{KARGER \\ Fax +4161306 1234 \\ E-Mail karger@karger.ch}

www.karger.com (c) 2011 S. Karger AG, Basel

1011-7571/11/0203-0253\$38.00/0

Accessible online at:

www.karger.com/mpp
Dr. Abiola C. Senok

College of Medicine, Alfaisal University

PO Box 50927

Riyadh 11533 (Saudi Arabia)

E-Mail asenok@alfaisal.edu 


\section{Introduction}

Gram-positive bacteria such as methicillin-resistant Staphylococcus aureus (MRSA), coagulase-negative staphylococci (MRCoNS) and vancomycin-resistant enterococci have emerged as important etiological agents in serious infections. Both MRSA and vancomycin-resistant enterococci (VRE) are now endemic in many health care institutions with an increased risk of patients being co-colonized by these two microorganisms. In vitro studies have shown that genes can be transferred from enterococci to S. aureus, Staphylococcus epidermidis, and other Gram-positive organisms via plasmid-mediated conjugation $[1,2]$. Thus, cross transfer of resistance genes from VRE to MRSA strains in such co-colonized patients represents a significant risk for the emergence of vancomycin-resistant $S$. aureus (VRSA). In fact vancomycin-resis$\tan S$. aureus reported so far arose due to the transfer of the vancomycin resistance determinant vanA from enterococcus to $S$. aureus [3]. These would potentially pose greater challenges in terms of treatment options and elimination strategies.

This study was carried out to determine the occurrence of VRE co-colonization among MRSA/MRCoNSpositive patients as well as determine the molecular fingerprinting of the MRSA isolates circulating in the hospital and the community.

\section{Materials and Methods}

\section{Setting and Patients}

The study was carried out at the Salmaniya Medical Complex (SMC), Kingdom of Bahrain over a 6-month period from November 2005 to April 2006. SMC serves as the main national secondary and tertiary referral center for specialist care, laboratory diagnosis and admissions. Ethical clearance was obtained from the Hospital Research and Ethics Committee and informed consent was given by all participants. Consecutive patients with cultureproven MRSA or MRCoNS colonization/infection during the study period were enrolled and stool specimens for VRE screening obtained. Specimens from two body sites (anterior nares and axilla) were obtained from close family contacts of these patients for MRSA/MRCoNS screening. Close family contacts were defined as those living in the same house or having prolonged contact with the patient. A total of 356 family members representing 113 families were enrolled in the study giving an average of 3 family members per patient. For 69 patients, we were unable to screen family members, because of refusal to participate or in some cases the family members were not domiciled in the country. Relevant demographic data, medical and surgical history for the preceding 6 months including previous hospitalization, antibiotic usage and presence of co-morbidities was obtained from patients and family members.

\section{Detection of MRSA/MRCoNS Isolates}

This was carried out using Diagnostic Susceptibility Test media (HiMedia Labs, India) supplemented with $4 \%$ (wt/vol) $\mathrm{NaCl}$ and containing $6 \mu \mathrm{g} / \mathrm{ml}$ oxacillin. Colonies showing characteristic staphylococcal morphology, microscopic appearance and positive catalase test were inoculated on Mueller-Hinton agar (Oxoid, Basingstoke, UK) supplemented with $5 \%$ sheep blood and incubated at $37^{\circ} \mathrm{C}$ for $24 \mathrm{~h}$. Colonies were confirmed to be $S$. aureus by positive tube coagulase test and DNase activity. Isolates negative for these tests were classified as MRCoNS. S. aureus strain ATCC33591 (oxacillin-resistant) was used as control. All MRSA isolates were confirmed by BD PHOENIX ${ }^{\mathrm{TM}}$ automated microbiology system (Becton Dickinson, USA). MRCoNS isolates were identified to species level using the API STAPH identification system (bioMérieux, Marcy l'Etoile, France) and BD PHOENIX ${ }^{\mathrm{TM}}$.

\section{Pulsed-Field Gel Electrophoresis}

To compare MRSA isolates obtained from the patients and their corresponding MRSA-positive family members, typing of isolates using pulsed-field gel electrophoresis (PFGE) was carried out at the Department of Microbiology, Kuwait University, Kuwait. Cells were treated as reported previously and digested with SmaI (Gibco BRL, Gaithersburg, Md., USA) according to the manufacturer's instructions [4]. Electrophoresis of SmaI-digested chromosomal DNA was performed using a CHEF-DRIII system (BioRad, Hercules, USA). A SmaI digest of S. aureus strain NCTC 8325 was used to normalize the gel as size standard [5]. Interpretation of gels was performed by visual inspection using the criteria of Tenover et al. [6]. The band patterns were compared and classified as indistinguishable (no band differences), closely related or clonal variants (one to three band differences) and subtypes of each other, possibly related (four to six band differences) and unrelated (more than six band differences) [6].

\section{Detection of VRE Isolates}

Stool specimens were inoculated directly on bile-esculin agar plates containing $0.15 \mathrm{~g} / \mathrm{l}$ sodium azide and incubated at $37^{\circ} \mathrm{C}$ for $48 \mathrm{~h}$ in ambient air. Enterococcus isolates were identified using standard microbiological methods and up to 10 colonies per plate were screened. Susceptibility to vancomycin was assessed by subculture of isolates on Mueller-Hinton agar containing $6 \mu \mathrm{g} / \mathrm{ml}$ vancomycin (Gulf Pharmaceutical Industries, United Arab Emirates), incubated at $37^{\circ} \mathrm{C}$ for $48 \mathrm{~h}$. Enterococcus faecalis ATCC29212 (vancomycin-sensitive) and E. faecalis ATCC51299 (vancomycinresistant) were used as controls. VRE isolates were identified to species level using API 20 STREP (bioMérieux) and BD PHOENIX $^{\mathrm{TM}}$. Vancomycin minimum inhibitory concentration as determined by BD PHOENIX ${ }^{\mathrm{TM}}$ was recorded. Isolates were preserved at $-80^{\circ} \mathrm{C}$ in stock culture trypticase medium (Becton Dickinson, USA) containing $20 \%$ glycerol until further analysis.

\section{Detection of Vancomycin Resistance Genotype by PCR}

Preserved isolates were thawed and inoculated on MuellerHinton agar. DNA for PCR was obtained from overnight cultures as follows: a colony was picked from the plate using a tooth pick and suspended in $400 \mu \mathrm{l}$ of TE buffer (10 mM Tris- $\mathrm{HCl}, 1 \mathrm{mM}$ EDTA, $\mathrm{pH} 8.0$ ), and the suspension was heated for $15 \mathrm{~min}$ at $100^{\circ} \mathrm{C}$, then centrifuged at $14,000 \mathrm{~g}$ at room temperature for $10 \mathrm{~min}$. The supernatant containing DNA was stored at $-20^{\circ} \mathrm{C}$ until further use. The genes for $\operatorname{van} \mathrm{A}$, vanB, vanC1, vanC2/C3, 
Fig. 1. Study flow chart.

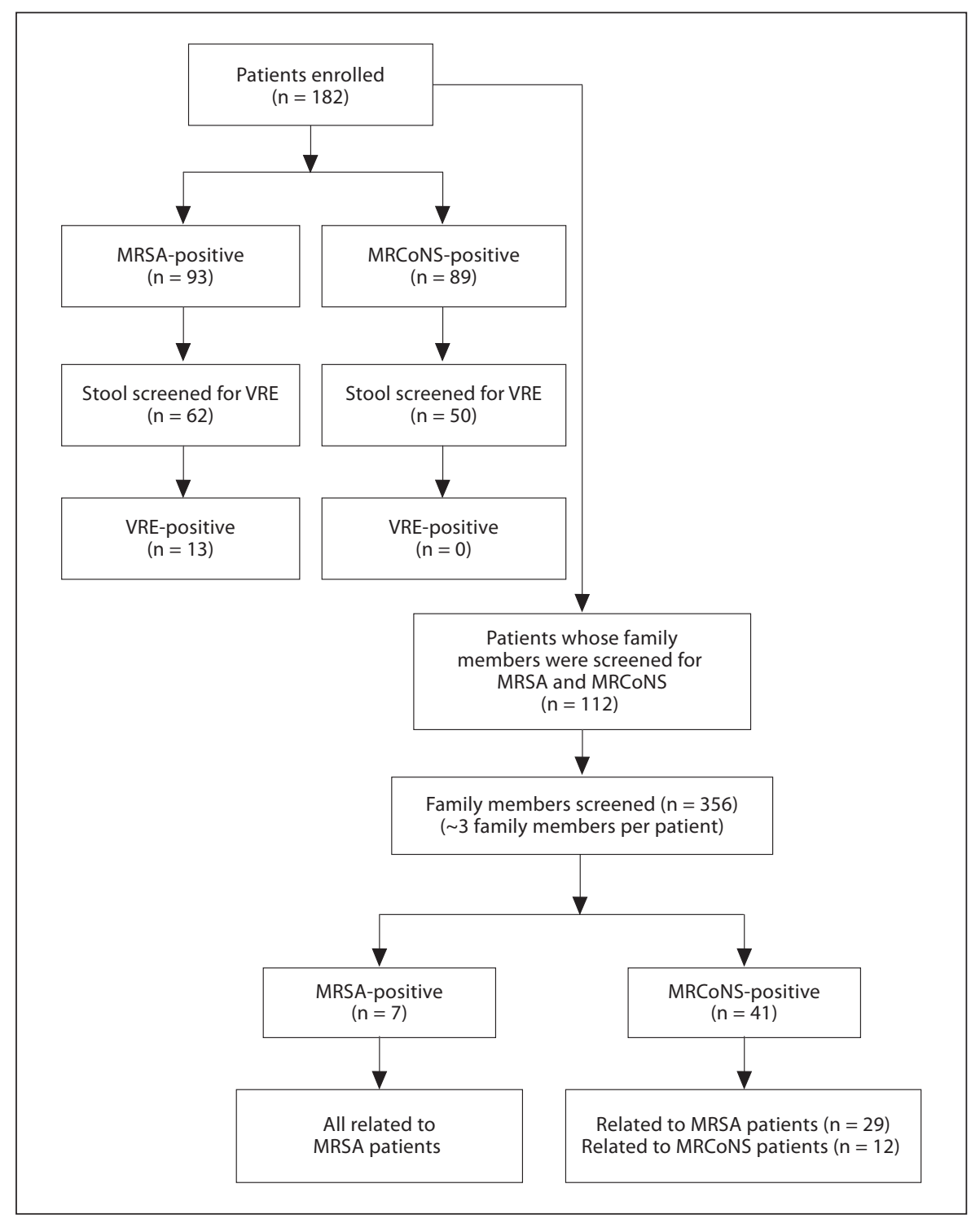

$d d l$ (Enterococcus faecium), and $d d l$ (E. faecalis) were amplified using previously described primers [7]. PCR was carried out with initial denaturation at $94^{\circ} \mathrm{C}$ for $5 \mathrm{~min}$, followed by 30 cycles of amplification (denaturation at $94^{\circ} \mathrm{C}$ for $1 \mathrm{~min}$, annealing at $54^{\circ} \mathrm{C}$ for $1 \mathrm{~min}$, and extension at $72^{\circ} \mathrm{C}$ for $1 \mathrm{~min}$ ), with a final extension at $72^{\circ} \mathrm{C}$ for $10 \mathrm{~min}$ in a GeneAmp ${ }^{\circledR}$ PCR system 9700 (PerkinElmer 9700, Applied BioSystem, Singapore). Gel electrophoresis was carried out using $15 \mu \mathrm{l}$ PCR products mixed with $1 \mu \mathrm{l}$ of the loading dye and run on a $1.2 \%$ agarose gel (Sigma Chemical Co. type 1-A, USA). A 100-bp DNA ladder (Bio-Rad EZ Load ${ }^{\mathrm{TM}}$, USA) was used as the molecular size marker.

\section{Statistical Analysis}

Data were analyzed with Statistical Package for Social Science (SPSS), version 13 . The $\chi^{2}$-test and Fisher's exact two-tailed test were used for statistical analysis.

\section{Results}

\section{MRSA/MRCoNS}

Of the 182 consecutive patients positive for MRSA/ MRCoNS infection or colonization enrolled in the study, male patients were $113(62 \%)$ and female 69 (38\%). The difference was statistically significant $(\mathrm{p}<0.01)$. Mean duration of hospitalization was 100 days and co-morbidity with chronic illnesses was present in 67 (37\%) patients. Of the 182 patients, 93 (51\%) had MRSA and 89 (49\%) had MRCoNS (fig. 1). The age range for MRSA patients was 4 weeks to 97 years (mean \pm SD: $55 \pm 21.4$ years) and for MRCoNS patients 10 days to 98 years (mean \pm SD: $59 \pm$ 19 years). 


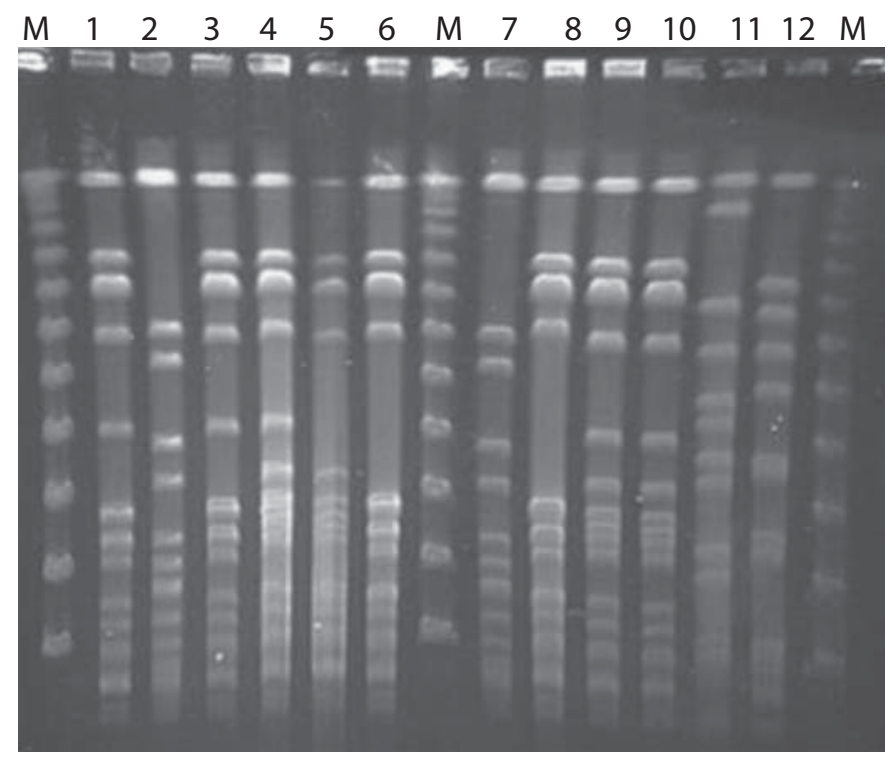

Fig. 2. PFGE of SmaI-digested total DNA from 12 MRSA strains. $\mathrm{M}=S$. aureus strain NCTC 8325 standard; lanes 1-5 = MRSA isolates from patients; lanes 6-12 = MRSA isolates from family members. Comparison of isolates from patient-family pairs: lane 1 vs. lane 6 , closely related or clonal variants; lane 2 vs. lane 7, indistinguishable; lane 3 vs. lane 8, closely related or clonal variants; lane 4 vs. lanes 9 and 10, indistinguishable; lane 5 vs. lanes 11 and 12 , unrelated.

The highest percentage of both MRSA and MRCoNS isolates were from the medical wards 35 (37.6\%) and 27 $(30.3 \%)$, respectively. In the pediatric ward and intensive care unit (ICU), the proportion of MRCoNS isolates (pediatrics 16, 18.0\%; ICU 18, 20\%) was significantly higher compared to MRSA (pediatrics 1, 1.1\%; ICU 8, $8.6 \%)$. In contrast in the burns unit, the MRSA isolates $(\mathrm{n} / \mathrm{N} \mathrm{14/93,15.1 \% )}$ were significantly higher compared to MRCoNS (n/N 3/89, 3.4\%; p < 0.05). For all other hospital wards, there was no significant difference in MRSA and MRCoNS isolates. Of 89 MRCoNS isolates 41 (46.1\%) were from blood which was significantly higher compared to $6.4 \%(\mathrm{n} / \mathrm{N}=6 / 93)$ for MRSA ( $\mathrm{p}<0.05$ ). In contrast for wound swabs the isolation of MRSA ( $\mathrm{n} / \mathrm{N}=39 / 93 ; 42 \%)$ was significantly higher compared to MRCoNs ( $/ \mathrm{N}=18 / 89 ; 20.2 \%$; $\mathrm{p}<0.05)$. There was no significant difference in the detection of MRSA and MRCoNS in other specimen types. However, all catheter tips yielded only MRCoNS.

From the 356 family members screened, 7 MRSA- and 41 MRCoNS-positive individuals were identified. The MRSA-positive family members were related to MRSA patients (fig. 1).

\section{PFGE Patterns}

The comparison of the banding patterns in patients with that of their corresponding family members is shown in figure 2 (lanes 1-5: MRSA isolates from patients, while lanes 6-12 MRSA isolates from family member). The patient-family pairs compared are as follows: lane 1 vs. lane 6 ; lane 2 vs. lane 7; lane 3 vs. lane 8 ; lane 4 vs. lanes 9 and 10; lane 5 vs. lanes 11 and 12 . This comparison of patient-family member pairs showed that isolates from 2 patients and their corresponding family members were indistinguishable (lane 2 vs. lane 7 and lane 4 vs. lanes 9 and 10, fig. 2). Isolates from 2 patients and their corresponding family members were related (lane 1 vs. lane 6 and lane 3 vs. lane 8). The MRSA isolate from the patient in lane 5 was not related to MRSA from the family members represented in lanes 11 and 12 .

\section{MRSA/VRE Co-Colonization}

Of the 112 patients (62 MRSA; 50 MRCoNS) who provided stool specimens for VRE screening, 13 (11.6\%) stool specimens were positive for VRE by PCR and all were obtained from MRSA-positive patients (fig. 1). The VRE positivity rate among the MRSA-positive cohort was $20.9 \%(n / N=13 / 62)$. A total of 14 isolates were characterized as 1 patient had two VRE isolates with different genotypes (Enterococcus gallinarum vanC and E. faecium vanB). The VRE isolates identified were $E$. faecium with vanB genotype $(\mathrm{n}=1)$; E. gallinarum with vanC1 genotype $(\mathrm{n}=11) ;$ E. gallinarum with vanC1 and vanB genotype $(n=2)$. The minimum inhibitory concentration for all isolates by the BD Phoenix system was $16 \mu \mathrm{g} / \mathrm{ml}$ (intermediate resistant). The majority of VRE isolates were from patients in medical and surgical units $(\mathrm{n} / \mathrm{N}=10 / 13$; $77 \%$ ). Male gender, prolonged hospitalization and presence of co-morbidities were significantly associated with MRSA/VRE co-colonization ( $\mathrm{p}<0.05)$.

\section{Discussion}

We have investigated the risk factors for MRSA/ MRCoNS infection, trafficking of MRSA between the hospital and the community as well as the occurrence of VRE co-colonization among patients at SMC Bahrain. The identified risk factors include gender, older age, hospital unit, duration of hospitalization and co-morbidity with chronic illnesses, similar to previous reports [8]. In many tertiary hospitals, the medical ward tends to be populated with older patients with chronic illnesses and prior prolonged stay at healthcare-related facilities. A 
similar scenario exists at SMC, which explains the high rates of isolation of MRSA and MRCoNS from the medical ward. Coagulase-negative staphylococci have been described as accounting for a large number of nosocomial bacteremias, especially those related to intravascular catheters as the bacteria are often carried from the site of insertion into the bloodstream [9]. The high rates of MRCoNS isolation in blood and catheter tips suggest that intravenous catheters are important sources of bacteremia in our setting.

To explore possible trafficking of MRSA between the community and the hospital environments the molecular fingerprinting of MRSA isolates from patients and their corresponding family member was carried out using PFGE. The findings indicate the presence of the same MRSA clone circulating in the hospital and the community. It also demonstrated the transmission of the same MRSA clones between patients and family members. Current evidence indicates that some MRSA clones were capable of persisting for long periods in hospitals and spreading among patients [10]. Additionally, familial transmissions of an MRSA clone have been described [11, 12]. A recent study from Bahrain has shown that the majority of MRSA isolates identified at SMC were persistent multiresistant PFGE clones with the SCCmec type III [13]. As none of the family members had a history of previous stay in a healthcare facility or any other risk factor for MRSA colonization, we speculate that patients could be the possible source of the MRSA trafficking into the community. This dissemination of MRSA from the hospital into the community poses a significant threat to public health as the family members serve as reservoirs of infection, thus undermining efforts at containing the spread of MRSA. However, as it could still be argued that MRSA trafficking occurs from the community to the hospital, we recommend further work on the molecular characterization of the MRSA isolates circulating in our setting.

Within this context of trafficking of MRSA between the hospital and the community, the greater problem is that of co-colonization with VRE in MRSA patients. It has been suggested that hospitalized patients with VRE colonization are at increased risk for MRSA colonization or infection and the clinical significance of this is highlighted by the emergence of VRSA [14, 15]. Our findings indicate a high prevalence of co-colonization with VRE among MRSA-positive patients particularly in the medical and surgical ward. It is of concern that $11.6 \%$ of all patients screened (20.9\% of the MRSA-positive cohort) were co-colonized with these pathogens, which is higher compared to the $2.7-9.5 \%$ in other studies [16-18]. Although no case of VRSA has yet been identified in our setting, the combination of similar risk factors for MRSA/ VRE colonization, high occurrence of co-colonization and the trafficking between the hospital and the community sets the stage for the possible emergence of VRSA within the hospital with the potential for its transfer into the community. It is interesting that no VRE co-colonization was observed in MRCoNS patients, the reason for this is unclear and we suggest further studies to clarify this finding. As the occurrence of VRE co-colonization among family members with MRSA colonization was not investigated, research work to assess co-colonization in the community and to determine factors that influence MRSA and VRE transmission in our setting is recommended.

In this study, the majority of VRE isolates (93\%) were E. gallinarum with vanC genotype and no vanA VRE was detected. This is significant as transfer of resistance determinants from chromosomally encoded vanC is extremely unlikely as opposed to plasmid-mediated transfer of vanA. The reason for the predominance of E. gallinarum with absence of $E$. faecalis in our population remains unclear. However, antibiotic therapy, particularly use of cephalosporins and vancomycin, has been suggested as being important in the upward trend of colonization with E. gallinarum [19]. In addition, a significant increase in the emergence of E. gallinarum in healthy subjects who were administered oral vancomycin has been reported [20]. Although E. gallinarum with vanC genotype had hitherto not been considered to be of public health significance because of their intrinsic resistance to glycopeptides and low pathogenicity, new evidence indicates that these organisms can cause serious invasive infections, and should be regarded as nosocomial pathogens $[21,22]$. Two of the E. gallinarum isolates identified in this study harbored the vanB gene as an additional resistance gene. A similar report of E. gallinarum isolates with the vanB gene as additional glycopeptide resistance gene has previously appeared in the literature and transfer of the vancomycin resistance genes between $E$. faecalis, E. gallinarum and Enterococcus casseliflavus has also been documented [22-25].

\section{Conclusion}

These data demonstrate high levels of co-carriage of MRSA and VRE among patients in a tertiary care facility with evidence suggestive of trafficking of MRSA between 
the hospital and community environment. This sets the stage for possible emergence of VRSA that could be disseminated into the community. Healthcare personnel should be cognizant of this possibility and appropriate methods for the early detection of co-colonized patients should be identified. In addition, measures to limit patient-to-patient transmission and prevent endemic colonization in the healthcare institutions and community should be implemented.

\section{References}

1 Perl TM: The threat of vancomycin resistance. Am J Med 1999;106:26S-37S.

-2 Zirakzadeh A, Patel R: Epidemiology and mechanisms of glycopeptide resistance in enterococci. Curr Opin Infect Dis 2005; 18: 507-512.

- 3 Tenover FC, Weigel LM, Appelbaum PC, McDougal LK, Chaitram J, McAllister S, Clark N, Killgore G, O’Hara CM, Jevitt L, Patel JB, Bozdogan B: Vancomycin-resistant Staphylococcus aureus isolate from a patient in Pennsylvania. Antimicrob Agents Chemother 2004;48:275-280.

4 Udo EE, Grubb WB: Genetic analysis of methicillin-resistant Staphylococcus aureus from a Nigerian hospital. J Med Microbio 1993;38:203-208.

5 Udo EE, Farook VS, Mokadas EM, Jacob LE, Sanyal SC: Molecular fingerprinting of mupirocin-resistant methicillin-resistant Staphylococcus aureus from a burn unit. Int J Infect Dis 1998;3:82-87.

6 Tenover FC, Arbeit RD, Goering RV, Mickelsen PA, Murray BE, Persing DH, Swaminathan B: Interpreting chromosomal DNA restriction patterns produced by pulsed-field gel electrophoresis: criteria for bacterial strain typing. J Clin Microbiol 1995;33: 2233-2239.

7 Dutka-Malen S, Evers S, Courvalin P: Detection of glycopeptide resistance genotypes and identification to the species level of clinically relevant enterococci by PCR. J Clin Microbiol 1995;33:24-27; erratum: J Clin Microbiol 1995;33:1434.

8 Safdar N, Maki DG: The commonality of risk factors for nosocomial colonization and infection with antimicrobial-resistant Staphylococcus aureus, enterococcus, gram-negative bacilli, Clostridium difficile, and Candida. Ann Intern Med 2002;136:834-844.

9 Rogers KL, Fey PD, Rupp ME: Coagulasenegative staphylococcal infections. Infect Dis Clin North Am 2009;23:73-98.
10 Udo EE, Jacob LE, Mathew B: The spread of a mupirocin-resistant/methicillin-resistant Staphylococcus aureus clone in Kuwait hospitals. Acta Trop 2001;80:155-161.

11 Huijsdens XW, van Santen-Verheuvel MG, Spalburg E, Heck ME, Pluister GN, Eijkelkamp BA, de Neeling AJ, Wannet WJ: Multiple cases of familial transmission of community-acquired methicillin-resistant Staphylococcus aureus. J Clin Microbiol 2006;44:2994-2996.

12 Urth T, Juul G, Skov R, Schonheyder HC: Spread of a methicillin-resistant Staphylococcus aureus ST80-IV clone in a Danish community. Infect Control Hosp Epidemiol 2005;26:144-149.

$\checkmark 13$ Udo EE, Panigrahi D, Jamsheer AE: Molecular typing of methicillin-resistant Staphylococcus aureus isolated in a Bahrain hospital. Med Princ Pract 2008;17:308-314.

14 Aligholi M, Emaneini M, Jabalameli F, Shahsavan S, Dabiri H, Sedaght H: Emergence of high-level vancomycin-resistant Staphylococcus aureus in the Imam Khomeini Hospital in Tehran. Med Princ Pract 2008;17:432434.

15 Appelbaum PC: The emergence of vancomycin-intermediate and vancomycin-resistant Staphylococcus aureus. Clin Microbiol Infect 2006;12(suppl 1):16-23.

16 Furuno JP, Perencevich EN, Johnson JA, Wright MO, McGregor JC, Morris JG Jr, Strauss SM, Roghman MC, Nemoy LL, Standiford HC, Hebden JN, Harris AD: Methicillin-resistant Staphylococcus aureus and vancomycin-resistant enterococci co-colonization. Emerg Infect Dis 2005;11:1539-1544.

17 Han SH, Chin BS, Lee HS, Jeong SJ, Choi HK, Kim CK, Kim CO, Yong D, Choi JY, Song YG, Lee K, Kim JM: Recovery of both vancomycin-resistant enterococci and methicillin-resistant Staphylococcus aureus from culture of a single clinical specimen from colonized or infected patients. Infect Control Hosp Epidemiol 2009;30:130-138.
18 Warren DK, Nitin A, Hill C, Fraser VJ, Kollef $\mathrm{MH}$ : Occurrence of co-colonization or coinfection with vancomycin-resistant enterococci and methicillin-resistant Staphylococcus aureus in a medical intensive care unit. Infect Control Hosp Epidemiol 2004;25:99104.

19 Reid KC, Cockerill IF, Patel R: Clinical and epidemiological features of Enterococcus casseliflavus/flavescens and Enterococcus gallinarum bacteremia: a report of 20 cases. Clin Infect Dis 2001;32:1540-1546.

20 Edlund C, Barkholt L, Olsson-Liljequist B, Nord CE: Effect of vancomycin on intestinal flora of patients who previously received antimicrobial therapy. Clin Infect Dis 1997;25: 729-732.

21 Corso A, Faccone D, Gagetti P, Togneri A, Lopardo H, Melano R, Rodriguez V, Rodriguez M, Galas M: First report of vanA Enterococcus gallinarum dissemination within an intensive care unit in Argentina. Int J Antimicrob Agents 2005;25:51-56.

22 Merquior VL, Goncalves Neves FP, Ribeiro RL, Duarte RS, de Andrade Marques E, Teixeira LM: Bacteraemia associated with a vancomycin-resistant Enterococcus gallinarum strain harbouring both the vanA and vanC1 genes. J Med Microbiol 2008;57:244245

23 Liassine N, Frei R, Jan I, Auckenthaler R: Characterization of glycopeptide-resistant enterococci from a Swiss hospital. J Clin Microbiol 1998;36:1853-1858.

-24 Dutka-Malen S, Blaimont B, Wauters G, Courvalin P: Emergence of high-level resistance to glycopeptides in Enterococcus gallinarum and Enterococcus casseliflavus. Antimicrob Agents Chemother 1994;38:16751677.

-25 Lu JJ, Wu JC, Chiueh TS, Perng CL, Chi WM, Lee WH: Characterization of a highly glycopeptide-resistant Enterococcus gallinarum isolate. J Formos Med Assoc 2000;99:305310 . 